\title{
Vertically Integrated Waveguide Polarization Splitters Using Polymers
}

\author{
Sean M. Garner, Vadim Chuyanov, Sang-Shin Lee, Antao Chen, \\ William H. Steier, Fellow, IEEE, and Larry R. Dalton
}

\begin{abstract}
Design considerations, fabrication procedures, and experimental results are presented for vertically integrated waveguide polarization splitters. These devices separate the TE and TM polarizations onto different vertical levels of a three-dimensionally integrated optic circuit. This provides increased integration density for polarization diverse structures. The vertical polarization splitters utilize birefringent polymer materials to create an adiabatic structure. Experimental results yielded extinction ratios as high as 22 and $12 \mathrm{~dB}$ for $\mathrm{TE}$ and TM polarizations, respectively.
\end{abstract}

Index Terms - Birefringence, integrated optics, optical interconnections, optical polymers, optical waveguides, waveguide bends, waveguide components.

\section{INTRODUCTION}

W AVEGUIDE polarization splitters separate the input light into two waveguide outputs; one for each orthogonal polarization. The splitting is typically in the horizontal plane for use in conventional two-dimensional (2-D) integrated optics. Vertical polarization splitters, on the other hand, split the polarization vertically into two different levels of stacked 2-D integrated optical circuits. They are therefore one of the basic components for achieving three-dimensional (3D) integrated optics. The 3-D integrated optics consist of vertically stacked layers of horizontal 2-D integrated optics with appropriately placed vertical waveguide interconnects between the layers. The third dimension significantly increases the possible density of both optical and electrical inputs and outputs. The stacked layers allow routing of waveguides around obstacles and eliminate waveguide cross-over losses. Vertical polarization splitters and the concept of 3-D integrated optics may have particular application in polarization diversity systems in which polarization insensitivity is achieved by providing two identical processing channels; one of each orthogonal polarization. In this approach, the two signal processing circuits are stacked vertically, and the vertical polarization splitter separates the random input polarization between the layers. This approach uses less substrate area and allows the

Manuscript received December 2, 1998; revised March 15, 1999. This work was supported by the Air Force Office of Scientific Research and by the Office of Naval Research.

S. M. Garner, V. Chuyanov, S.-S. Lee, A. Chen, and W. H. Steier are with the Department of Electrical Engineering-Electrophysics, University of Southern California, Los Angeles, CA 90089-0483 USA.

L. R. Dalton is with the Department of Chemistry, University of Southern California, Los Angeles, CA 90089-1062 USA.

Publisher Item Identifier S 1041-1135(99)05139-3.

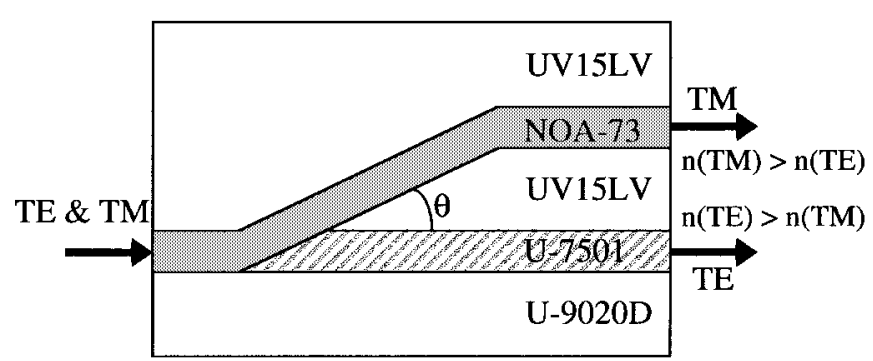

Fig. 1. General design and operation of vertical polarization splitter.

materials used in each of the 2-D layers to be optimized for that particular polarization.

\section{DESIGN}

Vertical polarization splitters act as adiabatic devices in which the input TE and TM modes couple to opposite vertical levels of the $Y$-branch output. Fig. 1 illustrates the general design and operation. Analogous to the horizontal case, vertical devices require the two polarizations to experience different effective indices in the two output branches. To fully separate the polarizations, one vertical layer must have a higher effective index for the TE mode, and the other layer must have a higher index for the TM mode. This causes the two polarizations to adiabatically transform into the fundamental mode of the output branch with the higher effective index [1].

Unlike horizontal $Y$-branches, the vertical fabrication of $Y$ branches offers the opportunity to easily make each branching arm out of completely different material. The branched structure of Fig. 1 incorporates birefringent polyimides (Ultradel 7501 and 9020D-Amoco) to define the lower waveguide and isotropic epoxies (NOA-73-Norland and UV15LV-MasterBond) to define the upper waveguide. At $\lambda=1.31 \mu \mathrm{m}, \mathrm{U}-7501$ has TE and TM refractive indexes of 1.562 and 1.526. U-9020D has TE and TM indexes of 1.522 and 1.495. Also, the isotropic NOA-73 and UV15-LV have indexes of 1.543 and 1.510 , respectively. We chose the epoxies because they have indexes that lie in between the polyimide birefringent values. In this way, the lower waveguide has a higher TE effective index, and the upper waveguide has a higher TM effective index. The polyimide U-9020D was selected for the lower cladding since it is compatible with the U-7501; its birefringence has only a small effect on the device operation.

The polarization extinction ratio at the output depends on the branching angle and the relative effective indexes of the two 


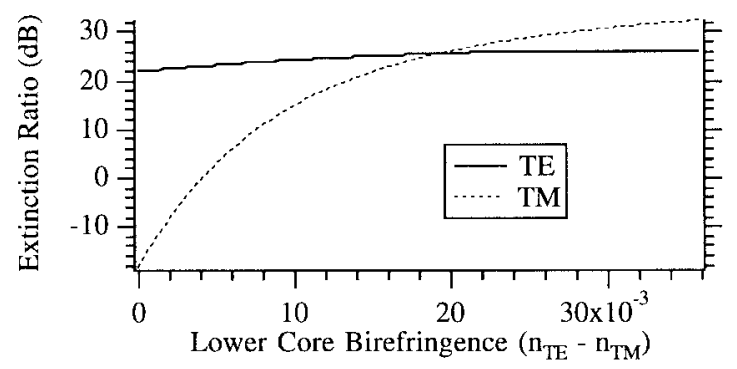

Fig. 2. Dependence of TE and TM extinction ratios on lower core birefringence. Simulation performed at $\lambda=1.31 \mu \mathrm{m}$.

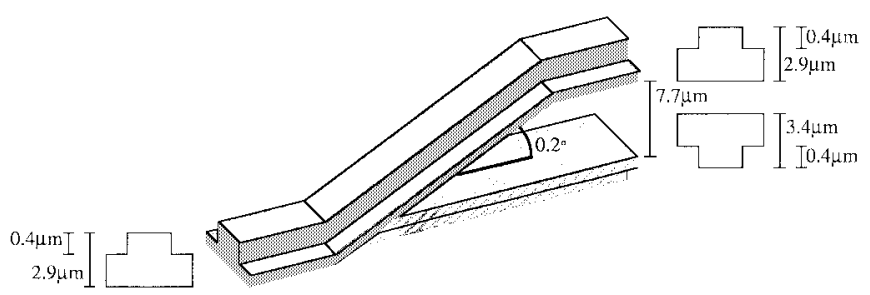

Fig. 3. Diagram of fabricated vertical polarization splitter.

output arms. The extinction ratio is defined as the power of a particular polarization in the expected output branch compared to the power of the same polarization in the other output. We performed 2-D beam propagation simulations to determine the optimum design. Fig. 2 shows the TE and TM extinction ratios dependence of the birefringence of the lower core material. The lower and upper core thicknesses were 3.5 and $3 \mu \mathrm{m}$, respectively, and the slope angle was $0.2^{\circ}$. The index of refraction of the lower core at zero birefringence was assumed to be 1.544 , the average of the two indices of Ultradel 7501. At very low values of birefringence both polarizations stay in the lower waveguide because of its higher effective index. At birefringence values $>0.02$, however, large extinction ratios are predicted for both polarizations. Ultradel 7501 has a TETM index difference of 0.0362 at $\lambda=1.31 \mu \mathrm{m}$ where Fig. 2 predicts polarization extinction ratios at the output of over 25 $\mathrm{dB}$ for both polarizations.

\section{FABRICATION}

The polarization splitters were fabricated on top of a $\mathrm{Si}$ wafer for a substrate. The procedure consisted of etching a lower channel waveguide in the lower cladding, spinning and curing the lower core and middle cladding, etching a vertical slope through the middle cladding and lower core, and finally, spinning and etching an upper ridge waveguide. Reactive ion etching (RIE) in oxygen is used to etch all of the features. The dimensions of the device are shown in Fig. 3. A dual-ridge/channel output waveguide design was fabricated to maximize the optical confinement throughout the structure. Reference [2] outlines the general fabrication procedure and specific slope etching techniques used to create similar vertical power splitters.

The different spin cast polymers required different curing procedures. The Ultradel polyimides required heating on a $150^{\circ}$ hot plate for 3-min immediately after spin casting. This provides good reflow smoothing of the films. Full curing of

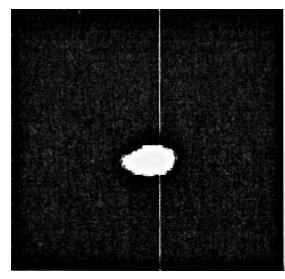

(a)

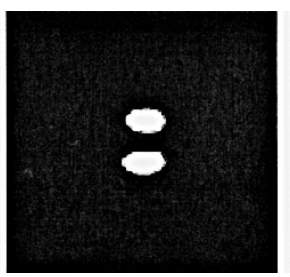

(b)

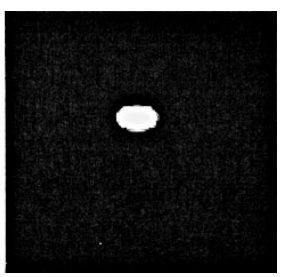

(c)
Fig. 4. Output of polarization splitter at $\lambda=1.31 \mu \mathrm{m}$ with analyzer aligned for: (a) TE, (b) TE/TM, (c) TM.

the polyimides took place in a $\mathrm{N}_{2}$ purged vacuum oven with a heating cycle of: $1 \mathrm{~h}$ at $80{ }^{\circ} \mathrm{C}, 2 \mathrm{~h}$ at $160{ }^{\circ} \mathrm{C}$, and $5 \mathrm{~h}$ at $200{ }^{\circ} \mathrm{C}$. This high temperature curing limits their use to only the lower layers in three-dimensionally integrated structures. Both epoxies used were UV cured with a 3-min exposure under a high-power light source (ILC Technology).

\section{EXPERIMENTAL RESULTS}

In the experimental setup, equal power in both the TE and TM polarizations at $\lambda=1.31 \mu \mathrm{m}$ was butt coupled from an $8-\mu \mathrm{m}$ core optical fiber into the waveguide. The waveguide outputs were collected with a $60 \times$ microscope objective lens, passed through a polarizer with $50-\mathrm{dB}$ extinction ratio, and focused onto the optical detector $50 \mathrm{~cm}$ away. This image size allowed a knife edge to block either the upper or lower waveguide outputs as needed. The fiber coupling was maximized by focusing the entire output pattern onto the detector, without passing through the analyzer, and then optimizing the fiber position.

Fig. 4 shows the output with different analyzer settings as viewed by an IR camera. Single-mode TE polarized output occurred from the lower waveguide, and the TM existed in the upper waveguide. No higher order modes appeared while varying the launch conditions of the input fiber.

The vertical polarization splitters demonstrated TE and TM output extinction ratios as high as 22 and $12 \mathrm{~dB}$, respectively, lower than predicted by the simulations and variable across the several splitters on the same substrate. We believe this is due to higher order and slab modes excited at the input and to scattering due to roughness in the etched vertical slope. The length of the input waveguide was $3 \mathrm{~mm}$ which may not be long enough for the higher order modes to dissipate before hitting the vertical taper and scattering into the wrong output waveguide. In simulation studies we found that higher order modes at the input could reduce the extinction ratios to $17 \mathrm{~dB}$ for both polarizations.

We obtained TE and TM insertion losses of $11.5 \pm 2.4 \mathrm{~dB}$ and $11.1 \pm 1.6 \mathrm{~dB}$, respectively. These losses compare the polarized output power from the correct waveguide branch with the power launched from the coupling fiber. Therefore, they include: fiber coupling into the waveguide, propagation loss through the material, effects of polarization extinction, and excess loss due to scattering. Because the input waveguide is both asymmetric and birefringent, the TE and TM modes have different vertical positions and mode shapes. A compromise, therefore, exists between optimum fiber coupling of the TE or 
TM waveguide modes. The higher insertion loss reflects this. Typical fiber coupling losses were estimated to be $5 \mathrm{~dB}$.

\section{CONCLUSION}

By utilizing the natural birefringent properties of polyimides, we designed and fabricated vertical polarization splitters. These structures launch the TE and TM modes into different vertical layers with experimental extinction ratios of about $15 \mathrm{~dB}$. The polyimides can operate at relatively high temperatures without any material degradation, and these vertical splitters should therefore have a larger useable temperature range than previously demonstrated polymer splitters [3] dependent on the alignment of chromophores. The hightemperature limit of the splitter we demonstrated is determined by the low $T_{g}$ UV epoxies and not the high temperature polyimides. However, we were able to demonstrate that these materials are stable to $150{ }^{\circ} \mathrm{C}$. After placing the NOA-73 and
UV15LV films on a $150{ }^{\circ} \mathrm{C}$ hot plate for $1 \mathrm{~h}$, the refractive index and thickness remained unchanged. After a temperature cycle of $200{ }^{\circ} \mathrm{C}$, however, the UV15LV index and thickness increased by 0.005 and $0.11 \mu \mathrm{m}$, respectively. By fabricating the entire polarization splitter out of isotropic and birefringent high temperature polyimides, the operating temperature can be expected to reach as high as $200{ }^{\circ} \mathrm{C}$.

\section{REFERENCES}

[1] W. K. Burns and A. F. Milton, "Mode conversion in planar-dielectric separating waveguides," IEEE J. Quantum. Electron., vol. QE-11, pp. 32-39, Jan. 1975.

[2] S. M. Garner, S.-S. Lee, V. Chuyanov, A. Chen, A. Yacoubian, W. H. Steier, and L. R. Dalton, "Three dimensional integrated optics using polymers," IEEE J. Quantum. Electron., to be published.

[3] M.-C. Oh, S.-S. Lee, S.-Y. Shin, W.-Y. Hwang, and J.-J. Kim, "Polymeric waveguide polarization splitter based on poling-induced birefringence," Electron. Lett., vol. 32, pp. 324-326, 1996. 